\title{
Evaluation of the Antifungal and Herbicidal Effects of Some Lamiaceae Plant Essential Oils in Invitro Conditions
}

\author{
Melih Yilar \\ Kirsehir Ahi Evran University, Faculty of Agriculture, \\ Department of Plant Protection, Kirsehir, Turkey \\ Yusuf Bayar \\ Kirsehir Ahi Evran University, Faculty of Agriculture, \\ Department of Plant Protection, Kirsehir, Turkey
}

\begin{abstract}
This study aimed to determine the herbicidal and antifungal activity of the essential oils of Rosmarinus officinalis L., Salvia absconditiflora Greuter \& Burdet and Thymbra spicata L.plants. In this study, plant essential oils against antifungal activity on Pyrenophora graminea plant pathogeni fungi and herbicidal effects on Taraxacum officinale were tested by using 0 (Control), $0.5,1,2,4 \mu \mathrm{L} /$ petri dish doses. The essential oils were applied by impregnation with a micropipette on filter paper adhered to the covers of petri dishes. As a result, the plant essential oils were found to be phytotoxic on Taraxacum officinale seed germination, seedling growth and Pyrenophora graminea mycelium growth. This plant essential oils are thought to be an alternative to synthetic pesticides in control of diseases and weeds.
\end{abstract}

Keywods: Herbicidal effects, Taraxacum officinale, antifungal effect, Pyrenophora graminea, essential oil

DOI: $10.7176 / \mathrm{JSTR} / 5-8-05$

\section{Bazı Lamiaceae Bitki Uçucu Yağlarının Antifungal Ve Herbisidal Etkilerinin İnvitro Koşullarda Değerlendirilmesi}

\begin{abstract}
Özet
Bu çalışmada, Rosmarinus officinalis L., Salvia absconditiflora Greuter \& Burdet. ve Thymbra spicata L. bitki uçucu yağlarının bazı herbisidal ve antifungal etkinliğinin belirlenmesi amaçlanmıştır. Elde edilen uçucu yağlar 0 (Kontrol), 0.5, 1, 2, $4 \mu \mathrm{L}$ /petri kabı dozunda bitki patojeni fungus Pyrenophora graminea üzerine antifungal etkisi ve Taraxacum officinale üzerine herbisidal etkisi test edilmiştir. Her iki uygulama içinde uçucu yağlar petri kabının üst kapağına yapıştırılan filtre kâğıdına mikropipetle emdirilerek uygulanmıştır. Sonuç olarak, bitki uçucu yağları artan dozla birlikte Taraxacum officinale tohum çimlenmesi, fide gelişimi ve Pyrenophora graminea miselyum gelişimi üzerine fitotoksik olduğu saptanmıştır. Bu bitki uçucu yağlarının hastalık ve yabancı otların kontrolünde sentetik pestisitlere alternatif olabileceği düşünülmektedir.
\end{abstract}

Anahtar Kelimeler: Herbisidal etki, Taraxacum officinale, antifungal etki Pyrenophora graminea, uçucu yă

\section{GİRIŞ}

Bitkisel üretimde hastalık, zararlı ve yabancı otlar farklı düzeylerde verim kayılarına neden olmaktadırlar. Bitki patojeni fungal hastalıklar hem üretim alanlarında hem de depolama aşamasında 
verim ve kalite kayıplarına yol açmaktadır. Yabancı ot; kültür bitkileri ile ışık, su, besin maddesi rekabete girmeleri bazılarının da sahip oldukları fitotoksik maddelerin olumsuz etkileriyle verim kayıplarına neden olabilmektedirler(Kong ve ark., 2007; Joshi and Joshi, 2016).Verim kayıpların önlenmesi için kullanılan sentetik pestisitlerin uzun yıllar bilinçsizce kullanılması patojenlerde ve yabancı otlara dayanıklılık problemi yanı sıra su, hava, toprak ve gidalarda toksik maddelerin birikmesi problemini beraberinde getirmiştir(Arslan ve Karabulut, 2005; Mengüç, 2018). Pestisitlerin bu olumsuz etkileri araştırmacıları yeni alternatif kontrol yöntemleri arayışlarına yöneltmiştir. Bu alternatif yöntemlerinden birisi kalıntı problemi olmayan hastalıkların ve yabancı ot kontrolünde kullanılabilecek doğal bileşiklerin belirlenerek uygulamalarda yer almalarının sağlanmasıdır.

Bitki hastalıkları ve yabancı otların kontrolünde kullanılabilecek doğal bileşiklerin başında da doğada kendiliğinden yetişen bitkilerin sahip oldukları sekonder bileşikler gelmektedir. Bu sekonder bileşiklerin antifungal, antibakterial, herbisidal, fitotoksik, insektisit,akarisit, nematisit, antioksidant gibi farklı biyolojik aktiviteye sahip olduğu çeşitli çalışmalarla ortaya konulmuştur(Kordali ve ark., 2005; Kordali ve ark., 2009; Bayar ve Yilar, 2019). Sekonder bileşiklerden uçucu yağlar bazı familyaya bağlı bazı türlerde baskın olarak bulunabilmektedir. Bu familyalardan olan Lamiaceae familyasının önemli gen merkezlerinden birisi ülkemiz olup familyanın 45 cinsi, 558 türü ve 742 taksonu mevcut olup endemizm oranı \% 42,2' dir (Koyuncu ve ark., 2010; Belen, 2012). Bu familyanın üyelerinden olan ve ülkemizde doğal olarak yetişebilen Biberiye (Rosmarinus officinalis L.), Zahter (Thymbra spicata L.) ve Kara şalba(Salvia absconditiflora Greuter \& Burdet.; Sin:Salvia cryptanthaMontbret \& Aucher ex. Benth.) herbisidal, antifungal, insektisit, akarisit, nematisit, antioksidan ve antimikrobiyal özellikleri yanı sıra geleneksel tıp, gıda ve ilaç endüstrilerinde kullanımı oldukça yaygın olan tıbbi ve aromatik bitkilerdir(Biljana ve ark., 2007; Gülbaba ve Özyurt, 2004; Bayan ve ark., 2017; Yılar ve Kadığlu, 2018).

Bu çalışma ile R. officinalis, T.spicata, S.absconditiflora bitki uçucu yağlarının fungal bir etmen olan arpa çizgi yaprak leke hastalığı(Pyrenophora graminea Ito. and Kuribayaski[= Helminthosporium gramineum (Rabenh.)]Eşeysiz dönemi, Drechslera graminea (Rabenh.) Shoemaker,) üzerine antifungal ve aslandişi(Taraxacum officinale) üzerine herbisidal etkinliğinin ortaya konması amaçlanmıştır.

\section{MATERYAL VE METOT}

2.1.Bitki materyalleri: Antifungal ve herbisidal testlerde kullanılan uçucu yağlar Çizelge 1'de verilen bitkilerden elde edilmiştir. Çiçeklenme aşamasında 2017-2018 yllında toplanan bitki materyalleri laboratuarda oda sıcaklığında gölgede kurutulmuştur. Kurutulan bitki materyalleri elektrikli değirmende öğütülmüşlerdir.

\begin{tabular}{|} 
Çizelge 1. Denemelerde kullanılan bitkiler \\
\begin{tabular}{|c|c|c|c|}
\hline $\begin{array}{c}\text { Bitkinin Latince } \\
\text { Adı }\end{array}$ & $\begin{array}{c}\text { Bitkinin } \\
\text { Türkçe Adı }\end{array}$ & $\begin{array}{c}\text { Bitkinin } \\
\text { Familyası }\end{array}$ & $\begin{array}{c}\text { Toplandı̆̆ } \\
\text { lokasyon }\end{array}$ \\
\hline Rosmarinus officinalis L. & Biberiye & Lamiaceae & İçel \\
\hline Thymbra spicata L. & Zahter & Lamiaceae & Kahramanmaraş \\
\hline $\begin{array}{c}\text { Salvia absconditiflora } \\
\text { Greuter \& Burdet }\end{array}$ & Kara şalba & Lamiaceae & Kırşehir \\
\hline
\end{tabular}
\end{tabular}

2.2. Uçucu yağların ekstraksiyonu: Bitkilerin uçucu yağları, Schilcher ${ }^{\circledR}$ cihazı kullanılarak hydrodistilasyon yöntemiyle elde edilmiştir. Bitki örneklerine saf su ilave edilerek $(1: 10 \mathrm{w} / \mathrm{v}) 2$ saat süre ile kaynatılmıştır. Elde edilen uçucu yağlar denemelerde kullanılana kadar $+4{ }^{\circ} \mathrm{C}$ 'de muhafaza edilmiştir(Telci ve ark., 2006).

2.3. Fungus kültürününelde edilmesi: Araştırma dakullanılan bitki patojeni Pyrenophora gramineaAhi Evran Üniversitesi Ziraat Fakültesi Bitki Koruma Bölümü Fitoklinik laboratuarlarında bulunan stok kültürden geliştirilmişstir. Bu stok kültürlerden; PDA içeren $90 \mathrm{~mm}$ petri kaplarında $25 \pm 2{ }^{\circ} \mathrm{C}$ 'de geliştirilmiş 7 günlük kültürler kullanılmıştır.

2.4. Uçucu yağların in vitro da Pyrenophora graminea' ya antifungal etkisi: 500 ml'lik erlanmayerlerde hazırlanan Patates Dekstroz Agar (PDA) ortamı otoklav edilmiş, $40^{\circ} \mathrm{C}$ 'ye kadar soğutularak $60 \mathrm{~mm}$ çaplı petri kaplarına yaklaşık $10 \mathrm{ml}$ olacak şekilde aktarılmıştır. Petri kaplarının kapaklarına steril kurutma kağıdı yapıştııılmış ve kurutma kağıtlarına mikropipet yardımı ile 0 (Kontrol), $0.5,1,2,4 \mu \mathrm{l} /$ petri dozlarında uçucu yağlar emdirilmiştir. Uygulama sonrası petri kapları parafilm ile 
kapatılmış ve $25^{\circ} \mathrm{C} \pm 2$ 'de inkübasyon ortamında tutulmuștur. 7 gün süren inkübasyondan sonra petri kaplarındaki miselyum gelişimleri dijital kumpas ile ölçülmüştür. Uygulamalar 4 tekerrürlü ve 2 tekrarlamalı olarak yürütülmüştür. Uçucu yağların \% engelleme oranı aşağıdaki eşitliğe göre hesaplanmıştır(Pandey ve ark., 1982).

\author{
MGI $\%=100 \times(\mathrm{K}-\mathrm{U}) / \mathrm{K}$ \\ MGI: \% Miselyum Gelişimi Engelleme Oranı \\ $\mathrm{K}$ : Kontroldeki radyal miselyum gelişimi \\ U: Uygulamalardaki radyal miselyum gelişimi
}

\title{
2.5. Uçucu yağların Taraxacum officinale tohum çimlenmesi ve fide gelişimine etkisi:
}

90 mm çaplı petri kaplarına 2 kat halinde steril kurutma kağıdı yerleştirilmiş ve Taraxacum officinale tohumlarından 25 adet sayılarak homojen olarak dağıtıllmıştır. Daha sonra kurutma kâğıtları distile su kullanılarak iyice nemlendirilmiştir. Petri kaplarının kapaklarına steril kurutma kağıdı yapıştırılmış, daha sonra bir mikropipet kullanılarak uçucu yağlar bu kağıt parçası üzerine damlatılarak, petri kapakları parafilm ile iyice kapatılmıştır(Önen ve ark., 2002). Denemede, uçucu yağlar 0 (Kontrol), 0.5, 1, 2, 4 $\mu 1 /$ petri dozlarda uygulanmıştır. Petri kapları 12 saat aydınlık-12 saat karanlık ve $24 \pm 2{ }^{\circ} \mathrm{C}$ (Kadioglu, 2004; Kordali ve ark., 2009) koşullarda 4 hafta süre ile inkübasyon ortamında tutulmuştur. Sürenin sonunda çimlenme oranı, kök ve sürgün boyları dijital kumpas yardımıyla ölçülmüştür. Denemeler 4 tekerrürlü ve 2 tekrarlamalı olarak yürütülmüştür.

2.6. İstatistiki analiz: Denemelerde muameleler arasındaki farkl1l1kların önem dereceleri varyans analizi (ANOVA) ile belirlenmiş, DUNCAN testi kullanılarak ortalamalar karşılaştırılmıştır. İstatistiksel analizler SPSS 15.0 bilgisayar programı kullanılarak yapılmıştır.

\section{BULGULAR VE TARTIŞMA}

Son yıllarda bitki uçucu yağlar ve ekstraktların biyolojik aktiviteleri üzerine yürütülen çalışmalar hız kazanmıştır. R.officinalis, T.spicata ve S.absconditiflora bitkilerinden elde edilen uçucu yağların Pyrenophora gramineamiselyum gelişimi ve Taraxacum officinale tohum çimlenmesi ve fide gelişimi üzerine fitotoksik etkisinin belirlenmesi için yürütülen çalışmada, farklı dozların etkinlikleri kontrollerle kıyaslanarak etki oranları hesaplanmıştır. Taraxacum officinale tohum çimlenmesi ve fide gelişimi uçucu yağlar farklı düzeylerde reaksiyon göstermiştir. Artan doza bağlı olarak fitotoksik etkide de artışlar meydana geldiği gözlenmiştir(Çizelge 2, Şekil 1).

Çizelge 2. Uçucu yağların Taraxacum officinale tohum çimlenmesi ve fide gelişimi üzerine olan etkisi

\begin{tabular}{|c|c|c|c|c|}
\hline Test Bitkileri & $\operatorname{Doz}(\mu \mathrm{l} /$ petri) & $\begin{array}{c}\% \\
\text { Çimlenme }\end{array}$ & $\begin{array}{c}\text { Kök } \\
\text { Uzunluğu } \\
\text { (cm) }\end{array}$ & $\begin{array}{c}\text { Sürgün } \\
\text { uzunluğu } \\
\text { (cm) }\end{array}$ \\
\hline \multirow{5}{*}{ R.officinalis } & Kontrol & $88.00 \pm 0.0^{\mathrm{a}}$ & $0.9 \pm 0.18^{\mathrm{a}}$ & $0.56 \pm 0.14^{\mathrm{a}}$ \\
\hline & 0.5 & $25.33 \pm 2.66^{\mathrm{b}}$ & $0.26 \pm 0.03^{b}$ & $0.30 \pm 0.0^{\mathrm{b}}$ \\
\hline & 1 & $18.66 \pm 3.52^{\mathrm{bc}}$ & $0.23 \pm 0.08^{\mathrm{b}}$ & $0.23 \pm 0.03^{\mathrm{b}}$ \\
\hline & 2 & $13.33 \pm 3.52^{\mathrm{cd}}$ & $0.16 \pm 0.08^{b}$ & $0.10 \pm 0.0^{\mathrm{b}}$ \\
\hline & 4 & $6.66 \pm 1.33^{\mathrm{d}}$ & $0.10 \pm 0.0^{\mathrm{b}}$ & $0.07 \pm 0.03^{\mathrm{b}}$ \\
\hline \multirow{5}{*}{ S. absconditiflora } & Kontrol & $88.00 \pm 0.0^{\mathrm{a}}$ & $0.9 \pm 0.18^{\mathrm{a}}$ & $0.56 \pm 0.14^{\mathrm{a}}$ \\
\hline & 0.5 & $21.33 \pm 1.33^{\mathrm{b}}$ & $0.36 \pm 0.18^{b}$ & $0.26 \pm 0.03^{\mathrm{b}}$ \\
\hline & 1 & $16.00 \pm 6.11^{\mathrm{bc}}$ & $0.023 \pm 0.12^{\mathrm{b}}$ & $0.13 \pm 0.00^{\mathrm{bc}}$ \\
\hline & 2 & $6.66 \pm 1.33^{\mathrm{cd}}$ & $0.10 \pm 0.00^{\mathrm{b}}$ & $0.10 \pm 0.03^{\mathrm{bc}}$ \\
\hline & 4 & $0.00 \pm 0.00^{\mathrm{d}}$ & $0.0 \pm 0.0^{\mathrm{b}}$ & $0.00 \pm 0.00^{c}$ \\
\hline \multirow{5}{*}{ T. spicata } & Kontrol & $88.00 \pm 0.0^{\mathrm{a}}$ & $0.9 \pm 0.18^{\mathrm{a}}$ & $0.56 \pm 0.14^{\mathrm{a}}$ \\
\hline & 0.5 & $33.33 \pm 5.81^{\mathrm{b}}$ & $0.13 \pm 0.08^{\mathrm{b}}$ & $0.13 \pm 0.08^{\mathrm{b}}$ \\
\hline & 1 & $12.00 \pm 2.30^{\mathrm{c}}$ & $0.13 \pm 0.03^{b}$ & $0.10 \pm 0.00^{\mathrm{b}}$ \\
\hline & 2 & $0.00 \pm 0.00^{\mathrm{d}}$ & $0.00 \pm 0.00^{\mathrm{b}}$ & $0.00 \pm 0.00^{\mathrm{b}}$ \\
\hline & 4 & $0.00 \pm 0.00^{\mathrm{d}}$ & $0.00 \pm 0.00^{\mathrm{b}}$ & $0.00 \pm 0.00^{\mathrm{b}}$ \\
\hline
\end{tabular}

*Aynı sütunda aynı harflerle ifade edilen ortalamalar arasındaki fark $\operatorname{ANOVA}(\alpha=0.005)$ göre önemli değildir.

R.officinalis uçucu yağı T.officinale tohum çimlenmesi ile kök-sürgün gelişimini artan doz bağlı olarak kontrole kıyasla istatistiki anlamda $\mathrm{p}<0.005$ önem düzeyinde olumsuz yönde etkilemiştir. $4 \mu 1 /$ petri

50 | P a g e

www.iiste.org 
dozda tohum çimlenmesini \%92.43, kök uzamasını \%90 ve sürgün uzamasını $\% 87.5$ oranında inhibe etmiştir. Diğer taraftan S.absconditiflora uçucu yağ $4 \mu \mathrm{l} /$ petri dozda, T.spicata uçucu yağ $2 \mu \mathrm{l} /$ petri dozda T.officinale tohum çimlenmesi ile kök-sürgün gelişimini tamamen inhibe etmişlerdir(Çizelge 1, Şekil 1).

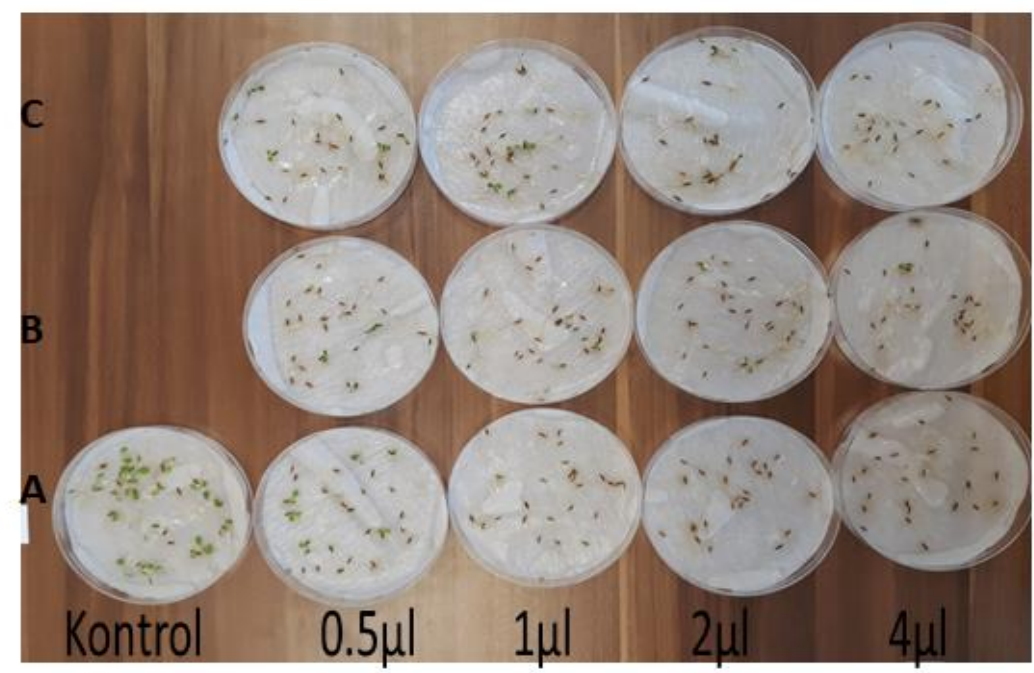

Şekil 1. Uçucu yağların Taraxacum officinale tohum çimlenmesi ve fide gelişimi üzerine olan etkisi(A:T.spicata,B:R.officinalis, C:S.absconditiflora)

Çizelge 3. Uçucu yağların Pyrenophora gramineamiselyum gelişimiüzerine olan etkisi

\begin{tabular}{|c|c|c|c|}
\hline Doz $(\boldsymbol{\mu l} /$ petri) & R.officinalis & T. spicata & S. absconditiflora \\
\hline Kontrol & $60.00 \pm 0.0^{\mathrm{a}}$ & $60.00 \pm 0.0^{\mathrm{a}}$ & $60.00 \pm 0.0^{\mathrm{a}}$ \\
\hline $\mathbf{0 . 5}$ & $53.16 \pm 1.30^{\mathrm{b}}$ & $5.33 \pm 0.00^{\mathrm{b}}$ & $42.00 \pm 2.64^{\mathrm{b}}$ \\
\hline $\mathbf{1}$ & $45.33 \pm 3.21^{\mathrm{bc}}$ & $0.00 \pm 0.00^{\mathrm{c}}$ & $35.00 \pm 0.76^{\mathrm{b}}$ \\
\hline $\mathbf{2}$ & $34.83 \pm 0.72^{\mathrm{cd}}$ & $0.00 \pm 0.00^{\mathrm{c}}$ & $34.66 \pm 1.09^{\mathrm{b}}$ \\
\hline $\mathbf{4}$ & $31.83 \pm 0.66^{\mathrm{d}}$ & $0.00 \pm 0.00^{\mathrm{c}}$ & $31.00 \pm 2.25^{\mathrm{b}}$ \\
\hline
\end{tabular}

*Aynı sütunda aynı harflerle ifade edilen ortalamalar arasındaki fark $\operatorname{ANOVA}(\alpha=0.005)$ göre önemli değildir.

Bitki uçucu yağlarının Pyrenophora graminea miselyum gelişimi üzerine etkili olduğu, bu etkinin uygulanan uçucu yağa, artan doza bağlı olarak değişkenlik gösterdiği belirlenmiştir. T. spicata uçucu yağ $1 \mu 1 /$ petri dozda miselyum gelişimini \%100 engellme oranı ile $P$. graminea üzerine yüksek derecede antifungal etki gösterebildiği, bunu sırasıyla $4 \mu \mathrm{l} /$ petri dozda S.absconditiflora ve $R$. officinalis izlediği belirlenmiştir(Çizelge 3, Şekil 2). Diğer taraftan $4 \mu 1$ petri dozunu hastalığın miselyum gelişimi tamamen engelleyemediği de belirlenmiştir.

Bitkilerde biyolojik aktivitelerin ortaya çıkmasını sağlayan sekonder bileşikler salgılayan bitkilerin canlıcansız dokularından buharlaşma, ayrışma, salınma gibi yollarla ortama verilmekte bu bileşiklerden biriside uçucu yağlardır. Bitki uçucu yağları bitki patojeni bakterileri kontak veya fumigant etki mekanizmalarıyla fungus, nematod, böcekler, akar ve yabancı otları kontak etki mekanizmasıyla öldürebilmektedir(Cunedioğlu ve Üremiş, 2018; Karakoç ve ark., 2018).

S.absconditiflora uçucu yağ ve ekstraktlarının çok faklı kültür bitkileri ve yabancı otlar üzerine biyoherbisidal; önemli bitki patojeni funguslar üzerine de antifungal etkiye sahip olduğu farklı çalışmalarla ortaya konmuştur(Yılar, 2014; Yılar ve ark., 2018). Lamiaceae familyası bitkisi olan Monarda didyma L. uçucu yağının doza bağlı olarak Taraxacum officinale tohum çimlenmesini engellediği Ricci ve ark.,(2017) tarafından rapor edilmiştir. Tworkoski(2002) 25 farklı bitkisel uçucu yağın Taraxacum officinale üzerine yürüttükleri etkinlik çalışmasında T.vulgaris, Satureja hortensis, Cinnamomum zeylanicum, Syzgium aromaticum uçucu yağlarının, hücre ölümlerine yol açtığını 
belirlemişlerdir. S.absconditiflora'ın dahil olduğu Salvia cinsine bağlı diğer türlerin de allelopatik etkiler sergileyebilmektedir(Kadığlu ve Yanar, 2004; Šućur ve ark., 2015; Mousavian ve Eskandari, 2018).

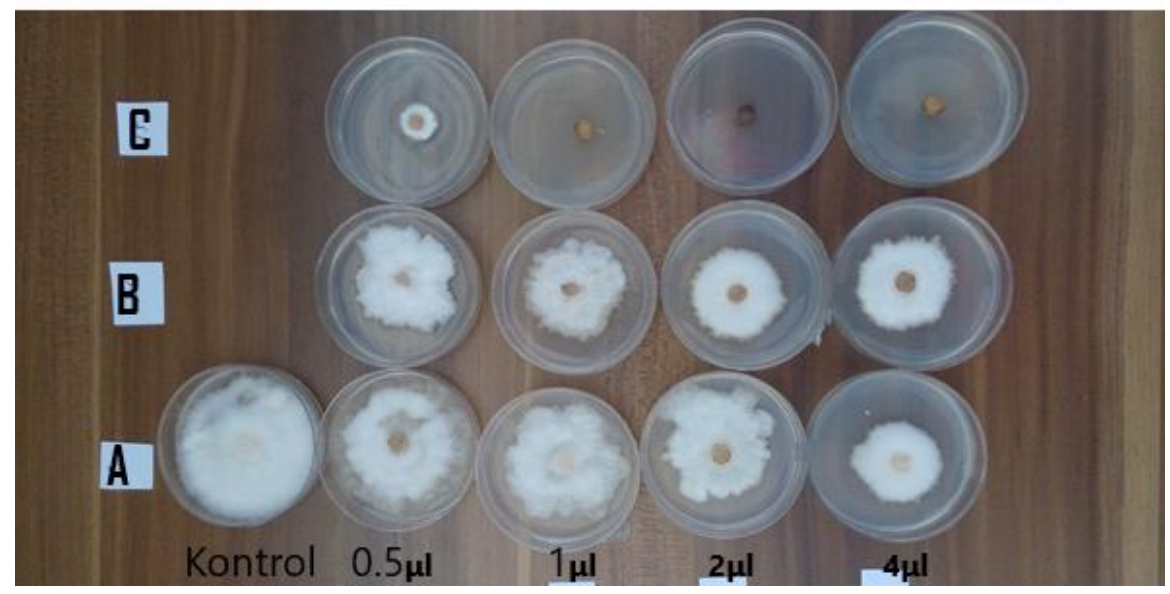

Şekil 2. Uçucu yağların Pyrenophoragramineamiselyum gelişimiüzerine olan etkisi(A:R.officinalis, $B:$ S.absconditiflora, $C:$ T.spicata)

R.officinalis bitki uçucu yağı fitotoksik etkiye sahiptir. Bitki uçucu yağı buğday genotipleri ve yabancı otlar üzerine allelopatik etki gösterdiği rapor edilmiştir(Atak ve ark., 2016). R. officinalis uçucu yağının Sorghum halepense gelişiminin değerlendirildiği bir çalışmada çıkış öncesi uygulamada \%41, çıkış sonrası uygulamada ise $\% 48$ oranında bitki gelişimini engellemiştir(Yazlık ve Üremiş, 2015). Thymbra spicata var. spicata uçucu yağının Triticum aestivum, Zea mays, Lactuca sativa, Lepidium sativum ve Portulaca oleracea bitkileri üzerine artan uygulama dozuna bağlı olarak fitotoksik etki gösterdiği belirlenmiştir(Ulukanli ve ark., 2016). R.officinalis, T.spicata ve benzeri bitkilerin yabancı otların mücadelesinde kullanılabilme potansiyelinin bulunduğu rapor edilmiştir(Özen ve ark., 2017).

Bazı bitkilerin allelopatik etkilerinın yanında bu bitkilerden elde edilen uçucu yağlar bitki patojeni fungusların mücadelesinde kullanılabilme potansiyeli bulunmaktadır. T. spicata ve R. officinalis uçucu yağının önemli bitki fungal patojeni Monilia fructigena miselyum gelişmesi üzerine fitotoksik etkiye sahip olduğu belirlenmiştir. Yürütülen bir çalışma da $R$. officinalis uçucu yağ $16 \mu 1 /$ petri dozunda, $T$. spicata uçucu yağ 1 ise $2 \mu \mathrm{l} /$ petri dozda miselyum gelişimini tamamen inhibe ettiği bildirilmiştir(Y (Ylar ve Bayar, 2018). Bitki patojeni funguslar üzerine S.absconditiflora uçucu yağ 1 ve ekstraktları fitotoksik etki göstermektedir. Bitki yağ 1 Fusarium oxysporum f. sp. radicis- lycopersici miselyum gelişimi üzerine etkiye sahiptir(Yilar ve Kadioğlu, 2016). Yine bu bitki uçucu yağının Sclerotinia sclerotiorum, Alternaria solani, Ascochyta rabiei, Botrytis cinerea, Rhizoctonia solani, Penicillum italicum, Aspergillus niger ve Monilia laxa patojenleri üzerine antifungal aktivitesi rapor edilmiştir(Yilar ve ark., 2018).

Sonuç olarak, endemik S. absconditiflora, T. spicata ve R. officinalis uçucu yağlarının invitro koşullarda herbisidal ve antifungal aktiviteye sahip olduğunu ve bu etkinin doza, uçucu yağ içeriğine bağlı olarak farklılık gösterdiği belirlenmiştir. Bitkisel üretimde artan sentetik pestisitlerin olumsuz etkilerinin azaltılması ve tarımın sürdürülebilmesinin yanı sıra çevrenin korunabilmesi için hastalık, yabancı otlar gibi bitki koruma konularında alternatif ve çevre dostu kontrol yöntemlerinin araştırılarak uygulamaya aktarılması bakımından bu ve benzeri çalışmalar önem arz etmektedir.

\section{Kaynaklar}

Arslan, Ü., Karabulut, Ö. A.2005. Baharat Bitkilerinin Bitki Patojeni Funguslara Karşı Antifungal Etkisi. Atatürk Üniv. Ziraat Fak. Dergisi, 36 (2), 131-135

Atak, M., Mavi, K., Uremis, I. 2016. Bio-herbicidal effects of oregano and rosemary essential oils on germination and seedling growth of bread wheat cultivars. Romanian Biological Letters, 21, (1), 11149-11159.

Bayan, Y., Genc, N., Kusek, M., Gul, F., Imecik, Z. 2017. Determinatıon of chemical compositions, antifungal, antibacterial and antioxidant activity of Thymbra spicata L. from Turkey. Fresen. Environ. Bull. 26, 7595-7599 
Bayar, Y. and Yilar, M., 2019. The Antifungal and Phytotoxic Effect of Different Plant Extracts of Salvia virgata Jacq. Fresenius Environmental Bulletin, 28(4A):3492-3497.

Belen, V., 2012. Farklı bölgelerden toplanan Salvia pilifera Montbet \& Aucher Ex Bentham populasyonlarının varyasyonları ve uçucu yağ bileşenleri açısından incelenmesi. (Yüksek Lisans Tezi). Kahramanmaraş Sütçü İmam Üniversitesi. Fen Bilimleri Enstitüsü, Biyoloji Anabilim Dal,, Kahramanmaraş.

Biljana, B., Mimica-Dukic, N., Samojlik, I., Jovin, E., 2007. Antimicrobial and antioxidant properties of rosemary and sage (Rosmarinus officinalis L. and Salvia officinalis L., Lamioceae) essential oils. Journal of Agricultural and Food Chemistry 55, 7879-7885.

Cunedioğlu, T., Üremiş, İ., 2018. Effects of Rosemary (Rosmarinus officinalis L.) and Turkish Oregano (Origanum minutiflorum O. Schwarz \& P.H. Davis) Essential Oils On Germination of Some Weed Seeds. Journal of Agricultural Faculty of Mustafa Kemal University, 23(1):24-32.

Gülbaba, A.G. ve Özyurt, N., 2004. Adana ve Mersin Yöresi Doğal Biberiye Populasyonlarının Alan, Yaprak ve Yağ Verimlerinin Belirlenmesi. 14. Bitkisel İlaç Hammaddeleri Toplantısı, Bildiriler, 29-31 Mayıs 2002, Eskişehir.

Kadioglu I.and Yanar Y., 2004. Allelopathic Effects of Plant Extracts Against Seed Germination of Some Weeds. Asian Journal of Plant Sciences, 3: 472-475.

Joshi, N. and Joshi, A., 2016. Allelopathic effects of weed extracts on germination of wheat. Annals of Plant Sciences 5.5: 1330-1334.

Kadioglu, I., 2004. Effects of Hearleaf Cocklebur (Xanthium strumariumL.) Extract on some crops and weeds. Asian Journal of Plant Sciences. 3 (6): 696-700.

Karakoç Ö.C, Alkan M., Şimşek Ş., Gökçe A., Çam H., 2018. Fumigant activity of some plant essential oils and their components against to Stegobium paniceum (L.) and Lasioderma serricorne (F.) (Coleoptera:Anobidae).. Plant Protection Bulletin, 58 (3). 163-169.

Kong CH, Zhao H, Xu XH, Wang P, Gu Y. 2007. Activity and allelopathy of soil of flavone Oglycosides from rice. J Agric Food Chem. 55:6007-6012.

Kordali, S., Çakır, A., Akcin, T.A., Mete, Akcin, E., Aydın, A.T. and Kılıç, H. 2009. Antifungal and herbicidal properties of essential oils and n-hexane extracts of Achillea gypsicola Hub-Mor. and Achillea biebersteinii Afan. (Asteraceae). Industrial Crops and Products. 29, 562-570

Kordali, Ş., Kotan, R., Mavi ,A., Çakır, A., Ala, A., Yıldırım, A., 2005. Determination of the Chemical Composition and Antioxidant Activity of the Essential Oil of Artemisia dracunculus and of the Antifungal and Antibacterial Activities of Turkish Artemisia absinthium Artemisia dracunculus Artemisia santonicum and Artemisia spicigera Essential Oils. Journal of Agricultural and Food Chemistry, 30;53(24):9452-8.

Koyuncu, O., Yaylacı, Ö.K., Öztürk, D., Erkara, İ.P., Savaroğlu, F., Akçoşkun, Ö. ve Ardıç, M., 2010. Risk categories and ethnobotanical features of the Lamiaceae taxa growing naturally in Osmaneli (Bilecik/Turkey) and environs. Biological Diversity and Conservation, 3(3), 31-45.

Mengüç, Ç., 2018. Herbicide Toxicity and Alternative Control Strategies Against to Weeds. Turkish Journal of Weed Science 21(1):61-73.

Mousavian, S.N., Eskandari, H., 2018. Allelopathic effect of two medicinal plants on seed germination, seedling growth and grain production of purslane (Portulaca oleraceae L.) weed. Acta agriculturae Slovenica, $111-2: 293-301$. 
Önen, H., Özer, Z. ve Telci, İ., 2002. Bioherbicidal effects of some plant essential oils on different weed species, Zeitschrift für Pflanzenkrankheiten und Pflanzenschutz. Journal of Plant Diseases and Protection, Sonderheft XVIII, 597-605.

Özen, F., Yaldız, G., Çamlıca, M., 2017. Allelopatic Effects of Some Aromatic Plants Essential Oils in Weed Control. International Journal of Agriculture and Wildlife Science (IJAWS), 3(1): 40 48 .

Pandey, D. K., Tripathi, N. N., Tripathi, R. D. and Dixit, S. N. 1982. Fungitoxic and phytotoxic properties of essential oil of Hyptis suaveolens. Z. Pflanzenkrankheiten Pflanzenschutz 89:344349.

Ricci, D., Epifano, F., Fraternale, D., 2017.TheEssential Oil of Monarda didyma L. (Lamiaceae) Exerts Phytotoxic Activity in Vitro against Various Weed Seed. Molecules, 22(2),222. E222. doi: 10.3390/molecules22020222.

Šućur, J., Popović, A., Petrović, M., Anačkov, G., Malenčić, D., Prvulović, D., 2015. Allelopathic Effects and Insecticidal Activity of Salvia sclarea L. STUDIA UBB CHEMIA, LX, 1:253-264.

Telci, I., Bayram, E., Yilmaz, G., Avci, B. 2006. Variability in essential oil composition of Turkish basils (Ocimum basilicumL.). Biochemical Systematics and Ecology. 34: 489-497.

Tworkoski, T., 2002. Herbicide effects of essential oils. Weed Science, 50:425-431.

Ulukanli, Z., Bozok, F., Cenet, M., Ince, H., Demirci, S.C., Sezer, G., 2016. Secondary Metabolites and Bioactivities of Thymbra spicata var. spicata in Amanos Mountains. Journal of Essential Oil Bearing Plants, 19 (7):1754-1761.

Yazlık, A., Üremiş, İ., 2015. Determination of the Efficiency of Some Essential Oil Compounds on the Development of Jonsongrass [(Sorghum halepense (L.) Pers.]. Turkish Journal of Agricultural Research, 2(2): 93-99.

Y1lar, M. 2014. Determination of antifungal and bioherbicidal activities of common Salvia species in Tokat Province. Gaziosmanpaşa University, Graduate School of Natural and Applied Sciences Department of Plant Protection, (Ph. D. Thesis), Tokat.

Y1lar, M. and Bayar, Y., 2018. Antifungal Activity of Thymbra spicata L. and Rosmarinus officinalis L. Essential Oils against Monilinia fructigena Honey in Whetze. Turkish Journal of Agricultural and Natural Sciences, 5(2): 121-126.

Yılar, M. and Kadığlu, İ., 2016. AntifungalActivities of some Salvia Species Extracts on Fusarium oxysporum f. sp.radicis lycopersici Mycelium Growth In vitro. Egyptian Journal of Biological Pest Control, Egyptian Journal of Biological Pest Control, 26(1), 115-118.

Yılar, M. and Kadığlu, İ., 2018. Salvia Species and their Biological Activities Naturally Distributed in Tokat Province. Sch. Bull., 4(2): 208-212.

Yilar, M., Kadioglu, I., Telci, I., 2018. Chemical Composition and Antifungal Activity of Salvia officinalis (L.), S. cryptantha (Montbret Et Aucher Ex Benth.), S. tomentosa (Mill.) Plant Essential Oils and Extracts. Fresenius Environmental Bulletin,27(3):1695-1706. 